\title{
Libérez le pharmacien!
}

\author{
par Glen Brown
}

$\mathrm{L}$ a complexité et la diversité des traitements médicamenteux Une cessent de croître, commandant ainsi un niveau de sophistication toujours plus poussé de la part des fournisseurs de soins. Si les pharmaciens veulent toujours avoir leur place en pratique contemporaine, ils doivent posséder les connaissances et les compétences leur permettant de reconnaître et de résoudre une large gamme de problèmes reliés à la pharmacothérapie. Cette exigence pour les pharmaciens de posséder des connaissances et des compétences étendues a entraîné des changements aux programmes d'enseignement des écoles de pharmacie et mené à l'établissement de programmes de formation et de rôles formels pour les praticiens de niveau supérieur. En revanche, les efforts consentis pour maximiser l'atout que représente le pharmacien dans les soins aux patients auront été vains si ces professionnels de la santé sont encombrés de tâches et de rôles qui ne requièrent pas leurs compétences particulières. Individuellement en tant que professionnels et collectivement en tant que services de pharmacie visant à maximiser la contribution des pharmaciens, nous devons déterminer et éliminer les activités et les tâches ne requérant pas nos compétences. En d'autres mots, il est temps de "libérer le pharmacien " de responsabilités et de rôles inopportuns.

En termes clairs, cela signifie que nous devons mettre fin à des fonctions spécifiques ou trouver une personne ou un automate pour s'en charger. Même s'il est plus efficient de cesser d'effectuer des tâches qui n'ont aucune valeur ajoutée, j'espère tout de même que la pratique actuelle est déjà centrée sur des activités essentielles et qu'il n'y a que peu d'occasions pour ce type de libération. Par conséquent, nous devons trouver des moyens de " sous-traiter " ces tâches à d'autres personnes ou à des automates.

Un groupe tout désigné pour jouer un rôle plus grand dans l'exécution de tâches ne faisant pas appel aux connaissances d'un pharmacien serait celui des techniciens en pharmacie. Dans ce numéro du JCPH, la chronique Le pour et le contre traite justement du plus grand rôle que sont appelés à jouer les techniciens en pharmacie en matière de planification et d'administration des systèmes de distribution des médicaments en établissements ${ }^{1,2}$. Les auteurs élaborent sur quelques-uns des avantages et des inquiétudes de ce transfert de responsabilités. J'invite tous les lecteurs à examiner les deux opinions et à se demander si cet élargissement du rôle des techniciens les aiderait à les libérer ou à libérer leurs collègues des tâches liées aux systèmes de distribution des médicaments.

Outre ce rôle potentiel que pourraient jouer les techniciens afin de libérer les pharmaciens pour qu'ils puissent passer plus de temps aux soins directs aux patients, de nombreux autres rôles novateurs pour les techniciens ont déjà été décrits dans des articles publiés par le $J C P H$, notamment un rôle de soutien aux soins directs aux patients prodigués par les pharmaciens. Par exemple, des auteurs de la chronique Le pour et le contre ont déjà discuté des avantages d'avoir des techniciens en pharmacie obtenir les histoires médicamenteuses et fournir des conseils aux patients au moment de leur congé, ${ }^{3,4}$. D'autres ont décrit le rôle que les techniciens pourraient jouer dans le bilan comparatif des médicaments, plus précisément dans l'obtention des meilleurs schémas thérapeutiques possibles 5 . La collecte des données nécessaires à l'établissement de la posologie optimale, avec la warfarine comme médicament prototype, a également été transférée aux techniciens et a donné d'excellents résultats ${ }^{6}$. Une autre réussite est celle de la collecte de données par un technicien au nom du pharmacien dans divers contextes cliniques (comme dans le cas de réactions indésirables aux médicaments, de conformité dans l'utilisation des médicaments, de traitements administrés en double $)^{7}$. Le transfert de ces rôles aux techniciens a permis aux pharmaciens de mieux utiliser leur temps et de se concentrer sur les activités de soins aux patients nécessitant leur expertise et leurs compétences.

De même, le recours à la technologie (principalement électronique) peut réduire le temps et les efforts nécessaires à l'obtention de l'information souhaitée pour reconnaître et résoudre les problèmes reliés à la pharmacothérapie. Tout comme les prescripteurs ont besoin de renseignements précis pour amorcer un traitement $t^{8}$, les pharmaciens ont besoin de renseignements pour évaluer le problème clinique d'un patient, déterminer les résultats souhaités, comparer les options thérapeutiques, établir un plan de suivi et informer le patient sur les problèmes reliés à la pharmacothérapie qu’il a éprouvés. 
Chacune de ces étapes peut demander du temps lorsqu'on doit chercher et extraire manuellement les données.

Imaginez le temps que pourrait économiser le pharmacien s'il pouvait simplement avoir accès à la base de données cliniques de l'établissement pour trouver toute l'information du dossier médical du patient, y compris les résultats d'analyses de laboratoire, les paramètres physiques contrôlés, les traitements médicamenteux en milieu communautaire (meilleur schéma thérapeutique possible) et les traitements actuels. Bien que la technologie permettant cela existe aujourd'hui, la plupart des établissements canadiens ne peuvent en assumer les frais. Par conséquent, jusqu’à ce que cette technologie soit largement répandue, les pharmaciens du Canada doivent examiner les façons d'utiliser les dispositifs électroniques qui pourraient les aider dans certaines de ces étapes. Par exemple, Fox et Felkey ont publié une série de chroniques dans Hospital Pharmacy, dans lesquelles ils décrivent de nouveaux dispositifs électroniques qui facilitent l'extraction de données pour les pharmaciens. Ils soulignent plus particulièrement le recours à des appareils mobiles permettant d'entrer de l'information sur les médicaments et les patients ${ }^{910}$. Des discussions au sujet d'avancées électroniques applicables à la pratique de la pharmacie ont cours dans des sites Web et des blogues ${ }^{11}$.

En transférant des tâches aux techniciens en pharmacie ou en ayant recours à des outils électroniques permettant de réduire le temps nécessaire à l'accomplissement de leurs tâches, les pharmaciens devraient être en mesure de " créer " du temps supplémentaire à consacrer aux activités de soins directs aux patients, qui nécessitent leur expertise et leurs compétences. Ce faisant, ils optimiseraient les bienfaits pour les patients. Nous devrions tous évaluer de façon critique notre pratique afin de déterminer les activités qui pourraient être transférées. Le temps est venu de libérer le pharmacien!

[Traduction par l'éditeur]

\section{References}

1. Raymond CB, Santos CE, Caligiuri C. Should pharmacy technician managers be responsible for the drug distribution system within hospital pharmacy departments? The "pro" side. Can J Hosp Pharm 2011; 64(3):218-219.
2. Levesque J. Should pharmacy technician managers be responsible for the drug distribution system within hospital pharmacy departments? The "con" side. Can J Hosp Pharm 2011;64(3):219-220.

3. Saulnier L. Should pharmacy technicians be trained to obtain medication histories and provide discharge counselling? The "pro" side. Can J Hosp Pharm 2008;61(6):441-442.

4. Schoen J. Should pharmacy technicians be trained to obtain medication histories and provide discharge counselling? The "con" side. Can J Hosp Pharm 2008;61(6):442-443.

5. Leung M, Jung J, Lau W, Kiaii M, Jung B. Best possible medication history for hemodialysis patients obtained by a pharmacy technician. Can J Hosp Pharm 2009;62(5):386-391.

6. Weber E, Hepfinger C, Koontz R, Cohn-Oswald L. Pharmacy technicians supporting clinical functions. Am J Health Syst Pharm 2005;62(23): 2466-2472.

7. Mark SM, Saenz R, Youich BE, Weber RJ. Innovative roles for pharmacy technicians: developing and implementing a unit-based clinical support pharmacy technician model. Hosp Pharm 2008;43(11):920-927.

8. Pollock M, Baaldua OV, Dobbie AE. Appropriate prescribing of medications: an eight step approach. Am Fam Physician 2007;15(2):231-236.

9. Felkey BG, Fox BI. Mobilizing care through portable information devices for patients and providers. Hosp Pharm 2010;45(10):801-802.

10. Fox BI, Felkey BG. Portable information appliances: an update. Hosp Pharm 2010;45(5):418-419.

11. Fox BI, Felkey BG. Getting value from blogs and other web resources. Hosp Pharm 2010;45(7):572-575.

Glen Brown, B. Sc. Pharm., Pharm. D., FCSHP, BCPS, est spécialiste en pharmacie clinique du Service de pharmacie du St Paul's Hospital de Vancouver, en Colombie-Britannique. II est également rédacteur adjoint du JCPH.

Adresse de correspondance :

Dr Glen Brown

Pharmacy Department

St Paul's Hospital

1081 Burrard Street

Vancouver BC V6H $1 \mathrm{G} 7$

e-mail: gbrown@providencehealth.bc.ca 